\title{
Effects of exposure to nanoparticle-rich diesel exhaust on 8-OHdG synthesis in the mouse asthmatic lung
}

\author{
MICHITAKA TANAKA $^{1}$, HIROHISA TAKANO ${ }^{2}$, YUJI FUJITANI ${ }^{3}$, SEISHIRO HIRANO ${ }^{3}$, \\ TAKAMICHI ICHINOSE ${ }^{4}$, AKINORI SHIMADA ${ }^{5}$ and KEN-ICHIRO INOUE ${ }^{1}$
}

\author{
${ }^{1}$ Center for Medical Science, International University of Health and Welfare, Ohtawara, Tochigi 324-8501; \\ ${ }^{2}$ Department of Environmental Engineering, Graduate School of Engineering, Kyoto University, Nishikyo-ku, \\ Kyoto 615-8540; ${ }^{3}$ Research Center for Environmental Risk, National Institute for Environmental Studies, \\ Tsukuba, Ibaraki 305-8506; ${ }^{4}$ Department of Health Science, Oita University of Nursing and Health Science, \\ Oita $871-1201 ;{ }^{5}$ Department of Veterinary Pathology, Tottori University, Tottori 680-8553, Japan
}

Received February 15, 2013; Accepted May 16, 2013

DOI: 10.3892/etm.2013.1198

\begin{abstract}
It has been demonstrated that exposure to diesel exhaust (DE) is associated with the induction and exacerbation of respiratory disorders; however, the impacts of DE containing mainly nanoparticles have been less studied. We have previously demonstrated that inhalation exposure to nanoparticle-rich DE (NR-DE) exacerbated allergic pulmonary inflammation, in the context of enhanced local expression of proinflammatory molecules. However, the underlying mechanisms have not been fully elucidated. 8-Hydroxydeoxyguanosine $(8-\mathrm{OHdG})$ is a marker of oxidative damage, particularly in DNA. This study examined the effects of NR-DE on 8-OHdG synthesis in the lung in the presence or absence of an allergen. Institute for Cancer Research (ICR) mice were exposed by inhalation to four different gas compositions (control air, low-concentration DE, high-concentration DE and high-concentration DE without particulate matter) for 8 weeks, in the presence or absence of repetitive intratracheal administration of ovalbumin (OVA). Thereafter, we assessed the levels of 8-OHdG synthesis and expression in the lungs by means of enzyme immunoassay (EIA) and immunohistochemistry. The EIA revealed that the level of $8-\mathrm{OHdG}$ was
\end{abstract}

Correspondence to: Professor Ken-Ichiro Inoue, Center for Medical Science, International University of Health and Welfare, 2600-1 Kitakanemaru, Ohtawara, Tochigi 324-8501, Japan

E-mail: kinoue@iuhw.ac.jp

Abbreviations: NR-DE, nanoparticle-rich diesel exhaust; 8-OHdG, 8-hydroxydeoxyguanosine; ROS, reactive oxygen species; PM, particulate matter; DEP, diesel exhaust particles; $\mathrm{PM}_{2.5}, \mathrm{PM}$ with a diameter $<2.5 \mu \mathrm{m}$; NOx, nitric oxides; SOx, sulphur oxides; PBS, phosphate-buffered saline; OVA, ovalbumin; BAL, bronchoalveolar lavage; BALF, BAL fluid; IL, interleukin

Key words: nanoparticle-rich diesel exhaust, allergic pulmonary inflammation, oxidative stress, $8-\mathrm{OHdG}$ significantly higher in the high-concentration NR-DE-exposed and allergen-sensitized/stimulated group compared with that in the control air-exposed and allergen-treated group. The immunohistochemistry results demonstrated that the level of immunoreactive 8-OHdG was higher in the NR-DE-exposed and allergen-treated lungs compared with that in the corresponding control air-exposed lungs. The results suggested that NR-DE exposure enhanced $8-\mathrm{OHdG}$ formation in asthmatic lungs. This, at least in part, is involved in the NR-DE-mediated exacerbation of the allergic pathophysiology that was identified in our previous study.

\section{Introduction}

The concentration of particulate matter (PM) with a mass median aerodynamic diameter (a density-dependent unit of measure used to describe the diameter of a particle) $\leq 2.5 \mu \mathrm{m}$ $\left(\mathrm{PM}_{2.5}\right)$ is more closely associated with respiratory effects and subsequent mortality than larger particles of mass median aerodynamic diameter $\leq 10 \mu \mathrm{m}\left(\mathrm{PM}_{10}\right)(1)$. A noteworthy aspect of the epidemiological data is that health impacts of $\mathrm{PM}_{2.5}$ are predominantly identified in subjects with predisposing factors to pneumonia, bronchial asthma, chronic obstructive pulmonary disease, compromised immune disorders and an age of $>65$ years (2). Diesel exhaust particles (DEPs), the main constituents of $\mathrm{PM}_{2.5}$ in urban areas, are epidemiologically considered to be harmful for respiratory systems and diseases (3). In accordance with this, the respiratory toxicity of DEPs has been biologically demonstrated, in the presence or absence of predisposing factors (4-6).

DEP sizes have become progressively smaller due to advancements in the automobile industry, leading to the production and release of particles $<100 \mathrm{~nm}$ in mass median aerodynamic diameter (defined as nanoparticles). This trend may increase the level of airborne nanoparticles, and consequently possess a greater health concern $(7,8)$. However, there have been few studies that have examined the effects of exposure to relevant types of nano-level DEPs on health, in individuals with or without predisposing factors. We have focused on nano- 
toxicity in allergic asthma. Since asthma is a chronic airway inflammatory disease and patients with asthma are reportedly highly sensitive to PM (9), we have demonstrated that inhaled nanoparticle-rich DE (NR-DE) exacerbated allergic airway inflammation in mice (10). The aggravation was concomitant with the amplified expression of the allergy-associated cytokines interleukin-5 (IL-5) and eotaxin, in the lung. However, the mechanisms for NR-DE-mediated aggravation of the allergic asthma model have not been fully investigated. The formation of 8-hydroxydeoxyguanosine $(8-\mathrm{OHdG})$ is the main DNA modification induced by reactive oxygen species (ROS) and may be responsible for DNA base mutations. It has been demonstrated that oxidative DNA adducts accumulate and are only repaired through enzyme pathways, resulting in further DNA damage (11). Oxidative DNA damage may be observed in lung inflammation, such as that induced by lipopolysaccharides (12). In addition, 8-OHdG expression is induced or enhanced in the lung as a result of several types of oxidative stress burdens, such as ozone (13), DEPs (14) or asbestos (15), in vitro and in vivo. In the present study, we investigated the levels of $8-\mathrm{OHdG}$ in the lung by means of enzyme immunoassay (EIA) and immunohistochemistry, to gain insights into the mechanistic pathway of the NR-DE-mediated aggravation of allergic airway inflammation.

\section{Materials and methods}

Animals. Female Institute for Cancer Research (ICR) mice (age, 6 weeks; weight, 29-33 g; Clea Japan, Inc., Tokyo, Japan) were used in this study. The mice were housed in an animal facility maintained at $24-26^{\circ} \mathrm{C}$ with $55-75 \%$ humidity and a $12 \mathrm{~h}$ light/dark cycle, and fed a commercial diet (Clea Japan, Inc.) with ad libitum access to water.

Generation of NR-DE inhalation systems. An 8-1 diesel engine (J08C; Hino Motors, Ltd., Hino, Japan) was used for generating the nanoparticles as previously described $(10,16)$. Four exposure chambers were set according to the conditions of the gases, and included a control (control air: CA), low-concentration $\left(36.3 \mu \mathrm{g} / \mathrm{m}^{3}\right.$ : D1) NR-DE, high-concentration $\left(168.8 \mu \mathrm{g} / \mathrm{m}^{3}\right.$ : D2) NR-DE and high concentration (168.8 $\mu \mathrm{g} / \mathrm{m}^{3}$ : D3) NR-DE without particulate components. In each inhalation chamber, the temperature and relative humidity were maintained at $20^{\circ} \mathrm{C}$ and $50 \%$, respectively.

Study protocol. The mice were exposed to one of the four different gas compositions (CA, D1, D2 and D3) in each chamber system for $5 \mathrm{~h} /$ day, 5 days a week for 8 weeks. During inhalation exposure, $1 \mu \mathrm{g} /$ body of ovalbumin (OVA) or vehicle [phosphate-buffered saline (PBS)] was intratracheally administered every 2 weeks (a total of five times). Finally, the mice were divided into eight groups, sacrificed and studied $24 \mathrm{~h}$ following the final intratracheal instillation ( 80 mice in total). The animal studies were approved by the Institutional Review Board of the National Institute for Environmental Studies, Tsukuba, Japan.

Bronchoalveolar lavage (BAL) procedure and 8-OHdG level in the BAL fluid $(B A L F)$. The mice were sacrificed by etherization and exsanguination from the abdominal aorta $24 \mathrm{~h}$ following the final intratracheal administration. A cannula was inserted into the trachea and secured with a suture. The lungs were lavaged three times with $1.2 \mathrm{ml}$ sterile saline at $37^{\circ} \mathrm{C}$, which was instilled bilaterally with a syringe. The fluid was harvested by gentle aspiration. The collected fluid was cooled and centrifuged at $300 \mathrm{x}$ g for $10 \mathrm{~min}$, as described previously (17-19). The collected supernatants were used for an EIA study ( $n=6$ in each group). In another experiment, the lungs were removed for immunohistological examination $(n=4$ in each group).

The EIA for determining the 8-OHdG level in the BALF was conducted on the basis of the competition between 8-OHdG and an 8-OHdG acetylcholine esterase conjugate (the 8-OHdG tracer) for a limited concentration of 8-OHdG monoclonal antibody. As the concentration of $8-\mathrm{OHdG}$ varies, the concentration of the tracer that is able to bind to the $8-\mathrm{OHdG}$ monoclonal antibody is inversely proportional to the $8-\mathrm{OHdG}$ level. Following incubation with the tracer, antibody and standard or sample in 96-well plates, the plates were washed to remove any unbound reagents, prior to Ellman's Reagent being added to the well. Finally, the product of this enzymatic reaction was read at $412 \mathrm{~nm}$ with conversion to $\mathrm{pg} / \mathrm{ml}$, using values obtained from the standard with limits of detection of $30 \mathrm{pg} / \mathrm{ml}$.

Immunohistochemistry. The degree of expression of 8-OHdG and its localization in the lungs were detected by immunohistochemistry using anti-8-OHdG monoclonal antibody (N45.1, Japan Institute for the Control of Aging, Nikken SEIL Co., Ltd., Fukuroi, Japan; n=4 in each group). The excised mouse lungs were embedded with paraffin. Following deparaffinization, the tissue sections were incubated with anti-8-OHdG antibody (dilution, 1:100) overnight at $4^{\circ} \mathrm{C}$, then reacted with biotinylated secondary anti-mouse $\mathrm{IgG}$ antibody (Vectastain Elite ABC kit; Vector Laboratories, Inc., Burlington, Canada) for $30 \mathrm{~min}$ at room temperature. Streptavidin was added and the color was developed with 3,3'-diaminobenzidine (DAB). Subsequently, the tissue sections were counterstained with hematoxylin (Merck, KGaA, Darmstadt, Germany) and examined by two researchers independently.

Statistical analysis. Data are presented as the mean \pm standard error. Differences between groups were determined using analysis of variance (the Student's t-test). $\mathrm{P}<0.05$ was considered to indicate a statistically significant difference.

\section{Results}

We first quantified the level of $8-\mathrm{OHdG}$ in the BALF. The level of 8-OHdG was higher in the D1- (299 pg/ml BALF), D2- (375 pg/ml BALF; P<0.05) and D3- (394 pg/ml BALF; $\mathrm{P}<0.05)$ OVA groups than in the corresponding vehicle groups (220, 160 and $186 \mathrm{pg} / \mathrm{ml}$ BALF, respectively), and was significantly higher in the D2- and D3-OVA groups than in the CA-OVA group (140 pg/ml BALF; P<0.05; Fig. 1A).

Subsequently, we investigated the expression levels and localization of $8-\mathrm{OHdG}$ in the lung specimens by means of immunohistochemistry. NR-DE plus OVA exposure induced moderate staining for $8-\mathrm{OHdG}$, compared with that of NR-DE alone or CA plus OVA exposure (Fig. 1B). The 

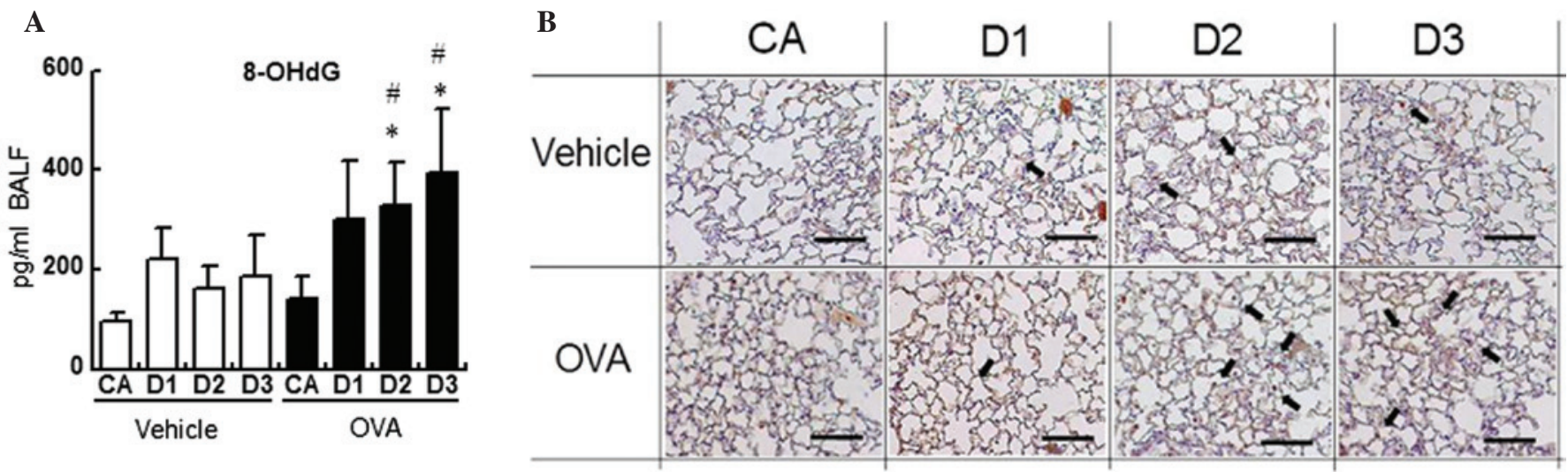

Figure 1. (A) Effects of inhalation exposure to nanoparticle-rich diesel exhaust (NR-DE) on 8-hydroxydeoxyguanosine (8-OHdG) levels in the bronchoalveolar lavage fluid (BALF). Institute for Cancer Research (ICR) mice were exposed to four patterns of gases [control air (CA); low-concentration diesel exhaust (DE; D1); high-concentration DE (D2); and high-concentration DE without particulate components (D3)] over a period of 8 weeks, and were simultaneously intratracheally administered vehicle or ovalbumin (OVA). BAL was performed $24 \mathrm{~h}$ following the final intratracheal administration, and the 8 -OHdG in the BALF was analyzed using an $8-\mathrm{OHdG}$ assay kit. Results are presented as the mean $\pm \mathrm{SE}\left(\mathrm{n}=6\right.$ in each group). ${ }^{*}<0.05$ vs. the corresponding vehicle group and ${ }^{\#} \mathrm{P}<0.05$ vs. CA-OVA. (B) Effects of inhalation exposure to NR-DE on 8-OHdG formation in the lung. Lungs were removed $24 \mathrm{~h}$ following the final intratracheal administration, then fixed and immunohistochemically stained using an anti-8-OHdG polyclonal antibody ( $\mathrm{n}=4$ in each group). Representative photomicrographs of the lung sections are shown. Arrows denote positive staining. Scale bar, $100 \mu \mathrm{m}$ (original magnification, x100).

8-OHdG expression was mainly localized to inflammatory polymorphonuclear leukocytes, such as neutrophils and eosinophils.

\section{Discussion}

Although DEPs $>200 \mathrm{~nm}$ in size have been demonstrated to induce adverse effects on several respiratory diseases, there have been few studies concerning the effects of DEPs containing mainly nanoparticles on lung pathology. Our previous study revealed that inhaled NR-DE exacerbated allergic airway inflammation in mice (10). The aggravation was concomitant with the enhanced expression of allergy-associated cytokines, interleukin-5 (IL-5) and eotaxin, in the lung. However, the mechanisms of the NR-DE-mediated aggravation of allergic asthma have not been fully investigated.

Oxidative stress, such as that due to ROS, is considered to be important in the pathogenesis of various types of lung inflammatory diseases, including allergic asthma $(20,21)$. In addition, a possible association between $\mathrm{PM}_{2.5}$ and oxidative stress has been identified. For example, environmentally relevant concentrations of $\mathrm{PM}_{2.5}$ have been demonstrated to exacerbate the airway inflammatory response with an increased generation of free radicals in asthmatic patients $(22,23)$. On the other hand, ROS may cause oxidative DNA modification, such as 8-OHdG formation (24). 8-OHdG is also induced by several types of oxidative stress-producing pollutants, such as ozone (13), DEPs (14) or asbestos (15), in vitro and in vivo. Concordant with these findings, it has been demonstrated that certain types of nanoparticles (carbon black nanoparticles and single/multi-wall nanotubes) were capable of increasing the expression of $8-\mathrm{OHdG}$ in the lung, in association with aggravated lung inflammation or injury $(19,25,26)$. In the present study, we quantified the level of 8-OHdG in the BALF. The level was greater in the D1-, D2- and D3-OVA groups than in the corresponding vehicle groups, and was significantly greater in the D2and D3-OVA groups than in the CA-OVA group $(\mathrm{P}<0.05)$.
Furthermore, the immunohistochemical analysis revealed that NR-DE plus OVA exposure induced moderate staining for 8-OHdG, compared with that of NR-DE alone or CA plus OVA exposure. However, no differences in staining were observed among the NR-DE plus OVA groups. These results suggested that NR-DE exposure increased 8-OHdG synthesis and release in the lung, which, at least in part, was involved in the NR-DE-mediated aggravation of the allergic pathophysiology that was identified in our previous study (10). The significant difference between the two parameters (EIA versus immunohistochemical staining) may be due to the time lag during translocation from the lung parenchyma to the bronchoalveolar spaces. Therefore, time-course studies may be required to increase understanding of the process.

Notably, gaseous components in the high-concentration NR-DE without particulate components (D3) significantly elevated the 8-OHdG levels in the BALF in the presence of allergen compared with CA $(\mathrm{P}<0.05)$. This is concordant with the allergic pathophysiology observed in the preceding study (10). 8-OHdG synthesis in the lung has been demonstrated to be induced or amplified as a consequence of DNA damage following exposure to gaseous pollutants, such as nitrogen oxides (NOx), sulphur oxides (SOx) or ozone $(23,27)$. Therefore, these gaseous components of NR-DE may be responsible for the enhanced formation of 8-OHdG. However, in the present study, particulate matter, NR-DEP, collected in the inhalation systems, independently elevated the 8-OHdG levels in the presence of OVA (data not shown), as we have previously identified in a study concerning nanoparticles (19). These results suggest that the mechanism of 8-OHdGhyperproduction may differ between high-concentration DE with and without particulate components.

NR-DE exposure significantly elevated the 8-OHdG level in the lung in the presence of an allergen (as compared with CA exposure). These results suggested that amplified 8-OHdG formation in asthmatic lungs, at least in part, is involved in the NR-DE-mediated aggravation of the allergic pathophysiology observed in our preceding study (10). 


\section{Acknowledgements}

The authors would like to thank Dr Masako Kiyono, Ryosuke Nakamura and Yuka Sone for their significant assistance. This study was supported by Grants-in-Aid for Scientific Research (B) 18390188 (to Ken-ichiro Inoue) from the Japan Society for the Promotion of Science, and partly by The Sumitomo-Foundation (Tokyo, Japan).

\section{References}

1. Peters A, Wichmann HE, Tuch T, Heinrich J and Heyder J: Respiratory effects are associated with the number of ultrafine particles. Am J Respir Crit Care Med 155: 1376-1383, 1997.

2. Dockery DW, Pope CA III, Xu X, et al: An association between air pollution and mortality in six U.S. cities. N Engl J Med 329: 1753-1759, 1993

3. Patel MM, Chillrud SN, Correa JC, et al: Traffic-related particulate matter and acute respiratory symptoms among New York City area adolescents. Environ Health Perspect 118 : $1338-1343,2010$.

4. Ichinose T, Furuyama A and Sagai M: Biological effects of diesel exhaust particles (DEP). II. Acute toxicity of DEP introduced into lung by intratracheal instillation. Toxicology 99: 153-167, 1995.

5. Takano H, Yoshikawa T, Ichinose T, et al: Diesel exhaust particles enhance antigen-induced airway inflammation and local cy tokine expression in mice. Am J Respir Crit Care Med 156: 36-42, 1997.

6. Maejima K, Tamura K, Nakajima T, et al: Effects of the inhalation of diesel exhaust, Kanto loam dust, or diesel exhaust without particles on immune responses in mice exposed to Japanese cedar (Cryptomeria japonica) pollen. Inhal Toxicol 13: 1047-1063, 2001.

7. Timonen KL, Hoek G, Heinrich J, et al: Daily variation in fine and ultrafine particulate air pollution and urinary concentrations of lung Clara cell protein CC161. Occup Environ Med 61: 908-914, 2004

8. Zhu Y, Eiguren-Fernandez A, Hinds WC and Miguel AH: In-cabin commuter exposure to ultrafine particles on Los Angeles freeways. Environ Sci Technol 41: 2138-2145, 2007.

9. Kappos AD, Bruckmann P, Eikmann T, et al: Health effects of particles in ambient air. Int J Hyg Environ Health 207: 399-407, 2004.

10. Tanaka M, Aoki Y, Takano H, et al: Effects of exposure to nanoparticle-rich or -depleted diesel exhaust on allergic pathophysiology in the murine lung. J Toxicol Sci 38: 35-48, 2013.

11. Kuchino Y, Mori F, Kasai H, et al: Misreading of DNA templates containing 8-hydroxydeoxyguanosine at the modified base and at adjacent residues. Nature 327: 77-79, 1987.

12. Kawai Y, Morinaga H, Kondo H, et al: Endogenous formation of novel halogenated 2'-deoxycytidine. Hypohalous acid-mediated DNA modification at the site of inflammation. J Biol Chem 279: 51241-51249, 2004.
13. Cheng ML, Ho HY, Huang YW, Lu FJ and Chiu DT: Humic acid induces oxidative DNA damage, growth retardation, and apoptosis in human primary fibroblasts. Exp Biol Med (Maywood) 228: 413-423, 2003.

14. Sanbongi C, Takano H, Osakabe N, et al: Rosmarinic acid inhibits lung injury induced by diesel exhaust particles. Free Radic Biol Med 34: 1060-1069, 2003.

15. Upadhyay D and Kamp DW: Asbestos-induced pulmonary toxicity: role of DNA damage and apoptosis. Exp Biol Med (Maywood) 228: 650-659, 2003.

16. Fujitani Y, Hirano S, Kobayashi S, et al: Characterization of dilution conditions for diesel nanoparticle inhalation studies. Inhal Toxicol 21: 200-209, 2009.

17. Inoue $\mathrm{K}$, Takano $\mathrm{H}$, Yanagisawa $\mathrm{R}$, et al: Pulmonary exposure to diesel exhaust particles induces airway inflammation and cytokine expression in NC/Nga mice. Arch Toxicol 79: 595-599, 2005.

18. Inoue K, Yanagisawa R, Koike K, et al: Effects of carbon black nanoparticles on elastase-induced emphysematous lung injury in mice. Basic Clin Pharmacol Toxicol 108: 234-240, 2010.

19. Inoue K, Yanagisawa R, Koike E, Nishikawa M and Takano H: Repeated pulmonary exposure to single-walled carbon nanotubes exacerbates allergic inflammation of the airway: Possible role of oxidative stress. Free Radic Biol Med 48: 924-934, 2010.

20. Riedl MA and Nel AE: Importance of oxidative stress in the pathogenesis and treatment of asthma. Curr Opin Allergy Clin Immunol 8: 49-56, 2008.

21. Li YJ, Takizawa H and Kawada T: Role of oxidative stresses induced by diesel exhaust particles in airway inflammation, allergy and asthma: their potential as a target of chemoprevention. Inflamm Allergy Drug Targets 9: 300-305, 2010.

22. Sierra-Vargas MP, Guzman-Grenfell AM, Blanco-Jimenez S, et al: Airborne particulate matter $\mathrm{PM}_{2.5}$ from Mexico City affects the generation of reactive oxygen species by blood neutrophils from asthmatics: an in vitro approach. J Occup Med Toxicol 4: 17, 2009.

23. Ren C, Fang S, Wright RO, Suh H and Schwartz J: Urinary 8-hydroxy-2'-deoxyguanosine as a biomarker of oxidative DNA damage induced by ambient pollution in the Normative Aging Study. Occup Environ Med 68: 562-569, 2011.

24. Kuwano K, Nakashima N, Inoshima I, et al: Oxidative stress in lung epithelial cells from patients with idiopathic interstitial pneumonias. Eur Respir J 21: 232-240, 2003.

25. Inoue $\mathrm{K}$, Takano H, Yanagisawa R, et al: Effects of nano particles on antigen-related airway inflammation in mice. Respir Res 6: $106,2005$.

26. Lai CH, Liou SH, Lin HC, et al: Exposure to traffic exhausts and oxidative DNA damage. Occup Environ Med 62: 216-222, 2005

27. Ren C, Fang S, Wright RO, Suh H and Schwartz J: Urinary 8-hydroxy-2'-deoxyguanosine as a biomarker of oxidative DNA damage induced by ambient pollution in the Normative Aging Study. Occup Environ Med 68: 562-569, 2011. 